\title{
ORIGINAL ARTICLE \\ Subacute T1-low intensity area reflects neurological prognosis for patients with cervical spinal cord injury without major bone injury
}

\author{
A Matsushita, T Maeda, E Mori, I Yugue, O Kawano, T Ueta and K Shiba
}

\begin{abstract}
Study design: A retrospective imaging and clinical study.
Objectives: To evaluate the relationship between magnetic resonance imaging (MRI) features and neurological prognosis in patients with traumatic cervical spinal cord injury (CSCl) without major bone injury.

Methods: A total of 72 patients with CSCI without major bone injury were treated conservatively in our hospital. MRI was performed for all patients at admission and 1 month following injury. We measured the antero-posterior and cranio-caudal diameter of intramedullary intensity changed area with T1-weighted images at the injured segment. Neurological evaluations were performed using the American Spinal Injury Association (ASIA) motor score and the modified Frankel grade at the time of admission and discharge.

Results: There was a significant relationship between the antero-posterior diameter ratio of the T1-weighted low-intensity area on MRI at the subacute stage and the ASIA motor score. The optimal threshold of the T1-weighted low-intensity diameter ratio for predicting the patient's ability to walk with or without assistance at discharge was determined to be $46 \%$. Moreover, $96.8 \%$ of the patients with $<50 \%$ T1-weighted low-intensity area recovered to walk with or without a cane at discharge.
\end{abstract}

Conclusion: The T1-low intensity area may be an important predictive factor for the neurological recovery of CSCI without major bone injury.

Spinal Cord (2016) 54, 24-28; doi:10.1038/sc.2015.84; published online 16 June 2015

\section{INTRODUCTION}

The incidence of cervical spinal cord injuries (CSCIs) without major bone injury has been increasing. Patients with these injuries show no evidence of fracture or dislocation of the spine on plain radiographs or computed tomography scans. ${ }^{1,2}$ The incidence, pathogenesis and severity of CSCI without major bone injury are different for different age groups because of the anatomical and biomechanical differences in the spine. ${ }^{3,4}$

Magnetic resonance imaging (MRI) is the best clinical tool for evaluating traumatic CSCI and is therefore invaluable for examining patients with CSCI without major bone injury. MRI can show the degree of spinal canal stenosis, as well as reveal the intramedullary state of the spinal cord in detail. ${ }^{5}$

To our knowledge, there have been only few reports on the MRI features of patients with CSCI without major bone injury. 6,7 The most common acute MRI pattern described is no change of signal intensity on T1-weighted images with a blurred high-intensity area on T2-weighted images. In the subacute and chronic stages, a low-intensity area begins to appear on T1-weighted images. The characteristic finding in the chronic stage is usually an oval-shaped area of signal change. ${ }^{8}$ On the basis of previous studies on the histopathological features of SCI, the blurred high-intensity area on T2-weighted images is thought to represent edema or petechial hemorrhage. On the other hand, the low-intensity area on T1-weighted images obtained in the subacute and chronic stages is thought to indicate necrosis, myelomalacia or an intramedullary cyst. ${ }^{5,9}$

Some papers have described the early relationship between MRI features and clinical outcomes in patients with CSCI at the acute stage following trauma. ${ }^{8,10,11}$ However, to the best of our knowledge, there are few papers reporting about this relationship at subacute or chronic stages following trauma.

In the present study, we evaluated T1- and T2-weighted MRI findings of patients with CSCI in the subacute stages. The purpose was to investigate the relationship between the MRI features and neurological prognosis of patients with CSCI without major bone injury at subacute and chronic stages.

\section{MATERIALS AND METHODS}

Patients with acute CSCI without major bone injury who were admitted to our hospital within 10 days after trauma were included in this study. The patients' imaging (radiographs, CT, MRI) was reviewed when they were admitted to our hospital. Small avulsion fractures of the vertebral body, spinous process fractures or bone bruises in the vertebral body without noticeable vertebral collapse were considered to be minor bony injuries. Any patient who had undergone previous cervical surgery and those who were likely to have recoil flexion injury were excluded, because of the artifacts from surgery implants in MRI. Recoil flexion injury requires fixation. It was difficult to do a correct evaluation in surgery cases. We also excluded patients who had no cord signal changes on MRI to rule out patients with cervical concussion or hysteria or those with a cord compression rate $>20 \%$. The rate of spinal cord 
compression was measured on sagittal MRI. The spinal cord diameter was measured at both the non-compressed and injured levels on T1-weighted MRI images, and the percent change was calculated by the following equation:

(antero-posterior diameter of the cord at no compression level-anteroposterior diameter of the cord at compression level)/antero-posterior diameter of the cord at no compression level $\times 100 \%$ (Figure 1). A value of $20 \%$ was defined as a cut-off for the spinal cord compression rate based on a previous report. ${ }^{12}$

From January 2005 to May 2010, 72 consecutive patients were included in this study. The mean patient age at the time of trauma was 62.4 years (34-81 years), and there were 60 men and 12 women. Using the American Spinal Injury Association (ASIA) impairment scale, paralysis at the time of admission was graded as $\mathrm{A}$ in 4 patients, $\mathrm{B}$ in 16, $\mathrm{C}$ in 43 and $\mathrm{D}$ in 9. The mean period from traumatic injury to admission was 1.5 days ( $0-10$ days), and the mean duration of hospital stay was 240.9 days (35-1423 days). All patients were treated conservatively and rehabilitated as quickly as possible with a simple neck brace.

MRI was performed in all patients at hospital admission (acute stage) and 1 month after trauma (subacute stage). Using sagittal MRI images, an intramedullary high-intensity changed area on T2-weighted MRI images was determined to be the injured level of the cervical spinal cord. We measured the antero-posterior diameter and cranio-caudal diameter of intramedullary intensity changed area on sagittal T1-weighted images (Figure 2). It was difficult to detect the intensity changed area on axial T1-weighted image. The measurement was taken at the largest spinal cord slice. The T1-weighted low-intensity changed area ratio (T1-LCAR) was calculated by the following equation: antero-posterior diameter of low-intensity changed area/spinal cord antero-posterior diameter $\times 100 \%$ (Figure 2 ).

All measurements were performed by two observers. Each measurement was performed three times, and the average value was calculated. All MR imaging examinations were performed with a 1.5-T magnet (MAGNETOM; Siemens Healthcare, Munich, Bayern, Germany). Standardized MR imaging protocols for the acutely injured spine were used: for sagittal T1-weighted imaging, a twodimensional spin-echo sequence was performed by using a conventional imaging option with no phase wrap, 525/11 (repetition time msec/echo time msec), a receiver bandwidth of $147 \mathrm{~Hz} / \mathrm{Px}$, a matrix of $320 \times 224$ (frequency encoding $\times$ phase encoding), three acquired signals and no phase correction. For sagittal T2-weighted imaging, a two-dimensional fast-recovery fast

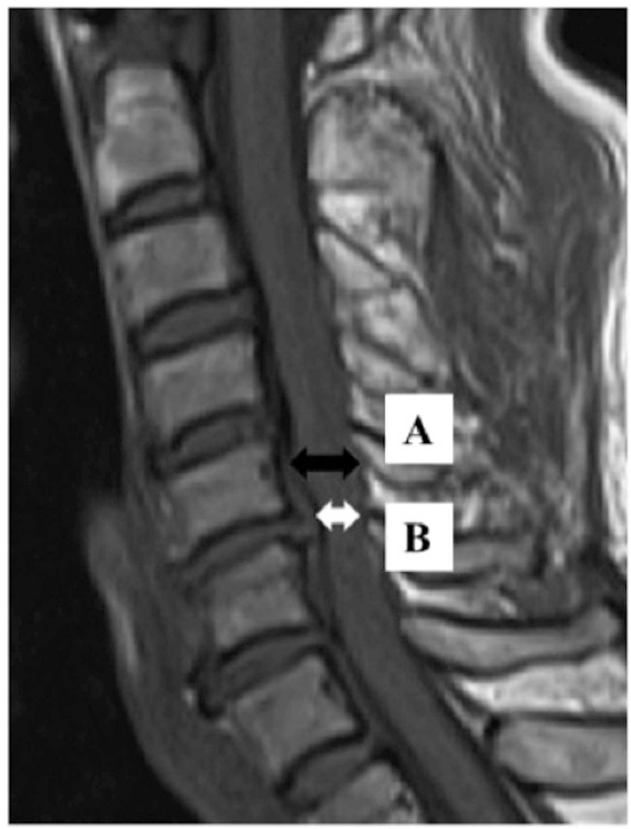

Figure 1 T1-weighted image. Black arrow (A) intact cervical spinal cord. White arrow (B) cervical spinal cord compressed by a disc and ligament flavum. Compression rate was 34\%. spin-echo (accelerated) sequence was performed by using imaging options that included the following: no phase wrap, an extended dynamic range, tailored radiofrequency and fast recovery; 3500/99; an echo train length of 15; a receiver bandwidth of $150 \mathrm{~Hz} / \mathrm{Px}$; a matrix of $384 \ldots 229$; four acquired signals; and phase correction. Both the T1- and the T2-weighted examinations were performed in the antero-posterior frequency direction by using an Neck Matrix coil, a section thickness of $3.0 \mathrm{~mm}$, an intersection gap of $0.3 \mathrm{~mm}$, a $24-\mathrm{cm}$ field of view and no contrast medium enhancement.

The ASIA impairment scale and the ASIA motor score (ranging from 0 to 100) were documented at admission and discharge for each patient. Neurologic recovery was evaluated as the following:

Improvement rate $(\%)=(($ motor score at discharge - motor score at admission $) /(100-$ motor score at admission $)) \times 100 .{ }^{13}$

All neurological evaluations were performed by senior spinal surgeons. In addition, we also documented each patient's score with the modified Frankel grading system (Table 1$).^{14,15}$

All data were analyzed using the JMP 8.0.2 software program (SAS Institute, Cary, NC, USA). The relationships between T1-LCAR and the ASIA motor scores and the recovery rate were analyzed using the Spearman rank-correlation coefficient. $P<0.05$ was considered statistically significant. The relationships between cranio-caudal diameter and the ASIA motor scores were also analyzed using the Spearman rank-correlation coefficient. The chance-corrected $\kappa$-coefficient was calculated to determine intra-observer agreement. Intra-observer reliability was almost good $(\kappa=0.92, P<0.001)$.

\section{RESULTS}

The neurologic statuses as evaluated by the ASIA and modified Frankel grading scales at admission and discharge are summarized in Tables 2 and 3. None of the patients demonstrated neurological deterioration during follow-up, and $60(81.9 \%)$ and 68 (94.4\%) demonstrated neurological recovery as evaluated by ASIA and modified Frankel grading, respectively.

The mean ASIA motor scores at admission and discharge were $37.3 \pm 29.2$ and $78.3 \pm 22.8$, respectively. The mean improvement rate of the motor score was $68.9 \pm 24.7 \%$, which was significant.

T1-weighted low-intensity changed areas were observed in 53 cases $(73.6 \%)$ at 1 month after injury. A total of 16 patients with final ASIA $\mathrm{D}$ and 3 patients with final ASIA E did not demonstrate intramedullary low-intensity change on T1-weighted MRI at the time of discharge. The T1-LCAR of these 19 patients was set as $0 \%$. The correlation between the T1-CAR and the ASIA motor score at discharge and the improvement rate were examined in all 72 patients, as shown in Figures 3 and 4. There was a significant negative correlation between T1-LCAR and ASIA motor score at discharge $(P<0.0001$, Figure 3$)$. There was also a significant negative correlation

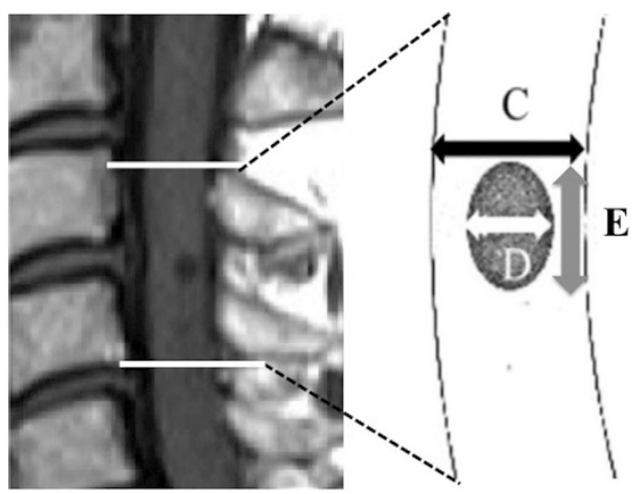

Figure 2 T1-weighted image. Black arrow (C) cervical spinal cord anteroposterior diameter. White arrow (D) T1-low antero-posterior diameter. T1-low diameter ratio was $\mathrm{D} / \mathrm{C}(\%)$. Gray arrow (E) T1-low cranio-caudal diameter (mm). 
Table 1 The modified Frankel grading system

\begin{tabular}{|c|c|}
\hline Grade & Neurological status \\
\hline A & Complete: no motor or sensory function \\
\hline B & Sensory only: some sensation preserved, no motor function \\
\hline B1 & Touch sensation remains in only sacral lesion \\
\hline B2 & Touch sensation remains in L/E \\
\hline B3 & Pain sensation remains in sacral lesion or $L / E$ \\
\hline C & $\begin{array}{l}\text { Motor useless: some sensory and motor function, but motor function not } \\
\text { useful }\end{array}$ \\
\hline $\mathrm{C} 1$ & Unable to flex the hip and knee from supine (Hip flexors 0-2) \\
\hline $\mathrm{C} 2$ & Able to flex the hip and knee from supine (Hip flexors 3-5) \\
\hline D & Motor useful: sensory function preserved, moter function weak but useful \\
\hline DO & $\begin{array}{l}\text { MMTs of } L / E \text { are } 4-5 \text {, but because of an acute phase, it is impossible to } \\
\text { test the walking ability }\end{array}$ \\
\hline D1 & Able to walk with a walker, but not practiced, usually use a wheel chair \\
\hline D2 & Independent gait with a cane \\
\hline D3 & Independent gait without a cane \\
\hline E & $\begin{array}{l}\text { Normal: normal sensory and motor function (hyperreflexia and numbness } \\
\text { are permitted) }\end{array}$ \\
\hline
\end{tabular}

Table 2 Diagram showing change in American Spinal Injury Association (ASIA) impairment scale between the status at admission (vertical axis) and at discharge (horizontal axis)

\begin{tabular}{|c|c|c|c|c|c|}
\hline $\begin{array}{c}\text { discharge } \\
\text { admission }\end{array}$ & A & B & C & D & E \\
\hline A & 1 & 0 & 3 & 0 & 0 \\
\hline B & 0 & 3 & 3 & 10 & 0 \\
\hline C & 0 & 0 & 2 & 39 & 2 \\
\hline D & 0 & 0 & 0 & 7 & 2 \\
\hline
\end{tabular}

between T1-LCAR and the recovery rate $(P=0.0072$, Figure 4$)$. On the other hand, there was no correlation between cranio-caudal diameter of T1 low-changed area and the ASIA motor score at discharge $(P=0.2797)$. There was also no correlation between cranio-caudal diameter of T1 low-changed area and the recovery rate $(P=0.2184)$.

A total of 60 patients $(83.3 \%)$ recovered to walk with or without a cane (higher or equal Frankel D). Receiver operator characteristic curve analysis demonstrated that the optimal T1-LCAR cut-off value for patients who were able to walk at discharge was $46 \%$ (Figure 5). If the T1-LCAR cut-off value was $<50 \%$, there was a significant positive correlation with being able to walk at discharge $(P<0.0001$, Pearson's $\chi^{2}$ test, Table 4).

\section{DISCUSSION}

The reported incidence of CSCI without major bone injury ranges from $\sim 10$ to $16 \%$ of all cervical cord injury in North America and India, ${ }^{6,16}$ but they are the largest proportion of cervical cord injury in Japan as described by Koyanagi et al. ${ }^{16}$ The number of CSCI without major bone injury has been increasing as the population ages; data from our institution showed an annual rate of 38.2\% in 1990 and $63.2 \%$ in $2005 .{ }^{17}$ CSCIs without major bone injury have consistently

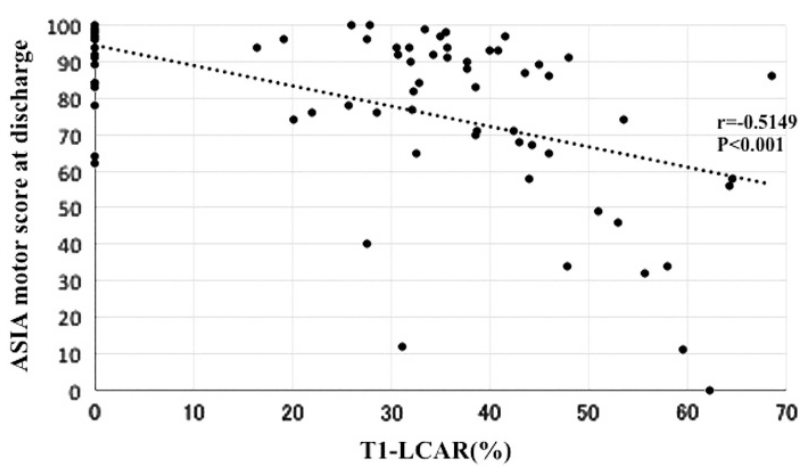

Figure 3 The relationship between the ASIA motor score at discharge and the T1-LCAR. There was a significant negative relationship between the two parameters, indicating that a larger T1-LCAR was associated with more severe paralysis.

Table 3 Diagram showing change in modified Frankel grade between the status at admission (vertical axis) and at discharge (horizontal axis)

\begin{tabular}{|c|c|c|c|c|c|c|c|c|c|c|}
\hline $\begin{array}{c}\text { discharge } \\
\text { admissiom }\end{array}$ & A & B1 & B2 & B3 & C1 & C2 & D1 & D2 & D3 & E \\
\hline A & 1 & 0 & 0 & 0 & 2 & 1 & 0 & 0 & 0 & 0 \\
\hline B1 & 0 & 0 & 2 & 0 & 1 & 0 & 2 & 2 & 0 & 0 \\
\hline B2 & 0 & 0 & 2 & 0 & 1 & 0 & 1 & 0 & 0 & 0 \\
\hline B3 & 0 & 0 & 0 & 0 & 1 & 0 & 2 & 2 & 1 & 0 \\
\hline C1 & 0 & 0 & 0 & 0 & 1 & 1 & 9 & 7 & 7 & 0 \\
\hline C2 & 0 & 0 & 0 & 0 & 0 & 0 & 0 & 7 & 4 & 0 \\
\hline D0 & 0 & 0 & 0 & 0 & 0 & 0 & 0 & 1 & 11 & 3 \\
\hline
\end{tabular}




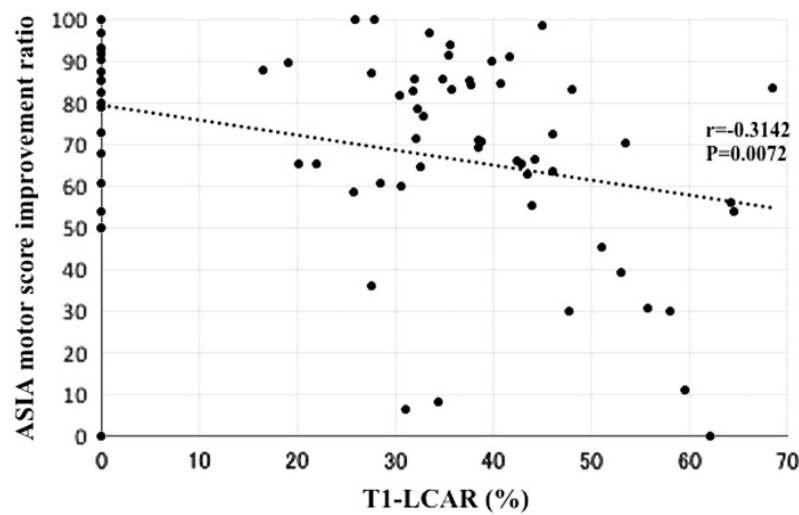

Figure 4 The relationship between the ASIA motor score improvement ratio at discharge and the T1-LCAR. There was a significant negative relationship between the two parameters, indicating that a larger T1-LCAR was associated with poor improvement.

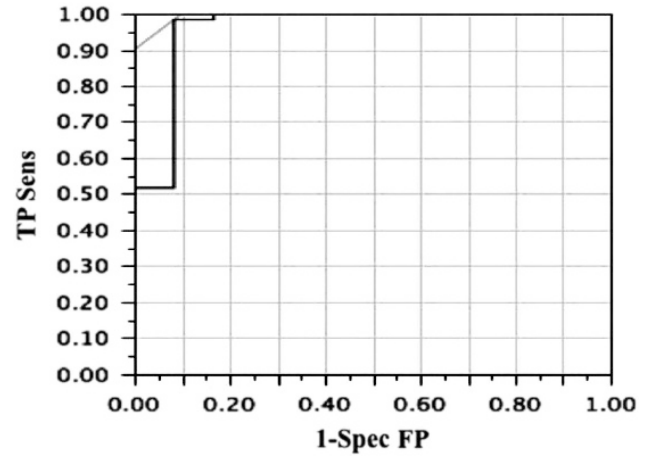

Figure 5 ROC curve of higher or equal Frankel grading D and T1-LCAR at 1 month after injury. AUC $=0.95833$. FP, false positive; Sens, sensitivity; Spec, specificity; TP, true positive.

been treated conservatively and rehabilitated as early as possible at our institution.

MRI is being increasingly used in the evaluation of post-traumatic myelopathy. It is useful in that it allows imaging of the injured cord, as well as an ability to predict patient outcome. The most common acute MRI pattern for CSCI is a blurred high-intensity area on T2-weighted images and no change in signal intensity on T1-weighted images. The subacute MRI pattern is iso-intensity or high-intensity on T2-weighted images, but a circumscribed low-intensity area is noted on T1-weighted images. This low-intensity area appears 3 to 6 weeks after injury. ${ }^{8}$ On the basis of previous studies of the histopathological features of SCI, the blurred high-intensity area on T2-weighted MRI is thought to represent edema or petechial hemorrhage. On the other hand, the low-intensity area on T1-weighted MRI in the subacute and chronic stages is thought to indicate necrosis or myelomalacia. ${ }^{5,9} \mathrm{We}$ thought that the T1-weighted low-intensity area on MRI at the subacute stage reflected the damage area of spinal cord. It was difficult to measure accurately the changed area of the spinal cord by edema in T2-weighted image. As there was no edema change in T1-weighted image, we could measure accurately the changed area of spinal cord.

Shimada and Tokioka ${ }^{18}$ identified four distinct patterns of MR signal intensity changes that correlated well with spinal cord damage severity and clinical outcome. Ishida and Tominaga ${ }^{19}$ assessed predictors of neurologic recovery in patients with acute central cervical SCI and found that an absence of MR signal intensity in the spinal
Table 4 T1-LCAR and modified Frankel grade at discharge. If the cut-off point for the T1-LCAR was $<50 \%$, a significant positive correlation was found between T1-LCAR $<50 \%$ and the patients who recovered to ASIA D or higher or to modified Frankel $D$ or higher $(P<0.0001)$

\begin{tabular}{|c|c|c|}
\hline & T1-LCAR $<\mathbf{5 0 \%}$ & T1-LCAR $>\mathbf{5 0 \%}$ \\
\hline Lower Frankel D & 2 & 10 \\
\hline $\begin{array}{c}\text { Higher or Equal } \\
\text { Frankel D }\end{array}$ & 60 & 0 \\
\hline
\end{tabular}

cord and good early neurologic improvement were important predictors of long-term neurologic function improvement. However, no studies have reported on T1-weighted low-intensity changed area in CSCI patients. We hypothesized that the T1-weighted low-intensity area on MRI at the subacute stage of injury was predictive of neurological outcome. Indeed, we identified a significant negative correlation between the T1-LCAR and the ASIA motor score at discharge. There was also a significant negative correlation between the T1-LCAR and the recovery rate. These results suggest that there might be significant correlation between the T1-weighted low-intensity changed area on MRI and post-traumatic neurological outcome. If the T1-LCAR was $<50 \%$, the patients achieved a neurological status higher or equal ASIA D or modified Frankel D. These patients were able to walk with or without a cane at discharge.

In this study, we researched the relationship between the outcome and the MRI 1 month after injury. All patients who did not have low-intensity area 1 month after injury were able to walk at discharge. Two months after injury, the low-changed area might be getting larger. However, this is the relationship between the outcome and the MRI 1 month after injury. MRI 1 month after injury reflects the patient's ability to walk.

This study had several limitations; it was retrospective, and the number of patients was small. Further research with larger patient populations and prospective evaluation may help resolve the questions raised in this study. In this study, the relationship between the imaging immediately after injury and the outcome was not performed. We did not consider the relationship between the outcome and the transverse diameter, cross-sectional area and volume of signal alteration, which will be considered in future study. Moreover, the etiology of CSCI without major bone injury should be studied in more detail.

In conclusion, we identified a significant relationship between T1-weighted low-intensity areas on T1-weighted MRI 1 month following injury and neurological recovery prognosis at discharge. Low-intensity changed area on T1-weighted MRI may be an important predictive factor in the natural course of neurological recovery for CSCI.

\section{DATA ARCHIVING}

There were no data to deposit.

\section{CONFLICT OF INTEREST}

The authors declare no conflict of interest. 
1 Gupta SK, Rajeev K, Khosla VK, Sharma BS, Paramjit, Mathuriya SN et al. Spinal cord injury without radiographic abnormalities in adult. Spinal Cord 1999; 37: 726-729.

2 Shen H, Tang Y, Huang L, Yang R, Wu Y, Wang P et al. Applications of diffusionweighted MRI in thoracic spinal cord injury without radiographic abnormality. Int Orthop 2007; 31: 375-383

3 Pang D, Wilberger JE. Spinal cord injury without radiographic abnormalities in children. J Neurosurg 1982; 57: 114-129.

4 Bhatoe HS. Cervical spinal cord injury without radiological abnormality in adults. Neurol India 2000; 48: 243-248.

5 Ohshio I, Hatayama A, Kaneda K, Takahara M, Nagashima K. Correlation between histopathologic features and magnetic resonance images of spinal cord lesions. Spine 1993; 18: 1140-1149.

6 Tewari MK, Gifti DS, Singh P, Khosla VK, Mathuriya SN, Gupta SK et al. Diagnosis and prognostication of adult spinal cord injury without radiographic abnormality using magnetic resonance imaging: analysis of 40 patients. Surg Neurol 2005; 63: 204-209.

7 Kasimatis GB, Panagiotopoulos E, Megas P, Matzaroglou C, Gliatis J, Tyllianakis M et al. The adult spinal cord injury without radiographic abnormalities syndrome: magnetic resonance imaging and clinical findings in adults with spinal cord injuries having normal radiographs and computed tomography studies. J Trauma 2008; 65: 86-93.

8 Takahashi M, Harada $\mathrm{Y}$, Inoue $\mathrm{H}$, Shimada $\mathrm{K}$. Traumatic cervical cord injury at C3-4 without radiographic abnormalities: correlation of magnetic resonance findings with clinical features and outcome. J Orthop Surg 2002; 10: 129-135.

9 Weirich SD, Cotler HB, Narayana PA, Hazle JD, Jackson EF, Coupe KJ et al. Histopathologic correlation of magnetic resonance imaging signal patterns in a spinal cord injury model. Spine 1990; 15: 630-638.
10 Machino M, Yukawa Y, Ito K, Nakashima H, Kanbara S, Morita D et al. Can magnetic resonance imaging reflect the prognosis in patients of cervical spinal cord injury without radiographic abnormality? Spine 2011; 36: E1568-E1572.

11 Miyanji F, Furlan JC, Aarabi B, Arnold PM, Fehlings MG. Acute cervical traumatic spinal cord injury: MR imaging findings correlated with neurologic outcome-prospective study with 100 consecutive patients. Radiology 2007; 243: 820-827.

12 Kawano O, Ueta T, Shiba K, Iwamoto Y. Outcome of decompression surgery for cervical spinal cord injury without bone and disc injury in patients with spinal cord compression: a multicenter prospective study. Spinal Cord 2010; 48: 548-553.

13 Ishida $\mathrm{Y}$, Tominaga T. Predictors of neurologic recovery in acute central cervical cord injury with only upper extremity impairment. Spine 2002; 27: 1652-1658.

14 Frankel HL, Hancock DO, Hyslop G, Melzak J, Michaelis LS, Ungar GH et al. The value of postural reduction in the initial management of closed injuries of the spine with paraplegia and tetraplegia. I Paraplegia 1969; 7: 179-192.

15 Hayashi T, Kawano O, Sakai H, Ideta R, Ueta T, Maeda T et al. The potential for functional recovery of upper extremity function following cervical spinal cord injury without major bone injury. Spinal Cord 2013; 51: 819-822.

16 Koyanagi I, Iwasaki Y, Hida K, Akino M, Imamura H, Abe H. Acute cervical cord injury without fracture or dislocation of the spinal column. J Neurosurg 2000; 93: 15-20.

17 Okada S, Maeda T, Ohkawa Y, Harimaya K, Saiwai H, Kumamaru H et al. Does ossification of the posterior longitudinal ligament affect the neurological outcome after traumatic cervical cord injury? Spine 2009; 34: 1148-1152.

18 Shimada K, Tokioka T. Sequential MR studies of cervical cord injury: correlation with neurological damage and clinical outcome. Spinal Cord 1999; 37: 410-415.

19 Ishida Y, Tominaga T. Predictors of neurologic recovery in acute central cervical cord injury with only upper extremity impairment. Spine 2002; 27: 1652-1658. 